\title{
Receptive Field (RF) Properties of the Macaque Second Somatosensory Cortex: RF Size, Shape, and Somatotopic Organization
}

\author{
Paul J. Fitzgerald, John W. Lane, Pramodsingh H. Thakur, and Steven S. Hsiao \\ Zanvyl Krieger Mind/Brain Institute, Departments of Neuroscience and Biomedical Engineering, Johns Hopkins University, Baltimore, Maryland 21218
}

\begin{abstract}
The detailed structure of multidigit receptive fields (RFs) in somatosensory cortical areas such as the SII region has not been investigated previously using systematically controlled stimuli. Recently (Fitzgerald et al., 2004), we showed that the SII region comprises three adjoining fields: posterior, central, and anterior. Here we characterize the RF structures of the 928 neurons that were reported in that study using a motorized oriented bar that was indented into the 12 finger pads of digits $2-5$. Most $(81 \%)$ of the neurons were responsive to the oriented bar stimuli, and $81 \%$ of those neurons had RFs that spanned multiple digits. Furthermore, the RFs varied greatly in size, shape, and complexity. Some RFs contained only excitatory finger pads, some contained only inhibitory pads, and some contained both types of pads. A subset of the neurons (23\%) showed orientation tuning within one or more pads. The RFs spread across different digits more than within individual digits, and the responsive finger pads for a given neuron tended to cluster together within the hand. Distal and lateral finger pads were better represented than proximal and medial finger pads. Furthermore, neurons in the posterior, central, and anterior SII region fields contained different proportions of RF types. These results collectively indicate that most SII region neurons are selective for different stimulus forms either within single finger pads or across multiple pads. We hypothesize that these RFs represent the kernels underlying the representation of tactile shape.
\end{abstract}

Key words: somatosensory cortex; tactile; SII; orientation; receptive field; somatotopy

\section{Introduction}

The receptive fields (RFs) of macaque monkey peripheral nerve fibers that convey discriminative touch information from the hand are restricted to a single finger pad (Iggo, 1963; Talbot et al., 1968). Neurons in area 3b of primary somatosensory cortex (SI) have larger RFs, some of which respond well to simple tactile stimulus features such as oriented bars (Hyvarinen and Poranen, 1978a; Warren et al., 1986; DiCarlo and Johnson, 2000). Like the peripheral neurons, most area $3 \mathrm{~b}$ neurons have single-digit RFs (Paul et al., 1972; Hyvarinen and Poranen, 1978b; Iwamura et al., 1983a; Chapman and Ageranioti-Belanger, 1991; DiCarlo et al., 1998). The second somatosensory (SII) region, which receives input from SI (Friedman et al., 1980; Friedman et al., 1986; Pons et al., 1987, 1992; Burton et al., 1995; Disbrow et al., 2003), contains many neurons with multidigit RFs (Whitsel et al., 1969; Robinson and Burton, 1980a; Sinclair and Burton, 1993; Krubitzer et al., 1995), indicating that the SII region plays an integrative role in processing information across separate digits.

The structure present within such cortical multidigit RFs has not been investigated previously in detail. For example, the precise sizes and shapes of these RFs are not known. Moreover, it is

Received June 8, 2005; revised May 11, 2006; accepted May 12, 2006.

This work was supported by National Institutes of Health Grant NS34086.

Correspondence should be addressed to Dr. Steven Hsiao, Zanvyl Krieger Mind/Brain Institute, Johns Hopkins University, 338 Krieger Hall, 3400 North Charles Street, Baltimore, MD 21218. E-mail: steven.hsiao@jhu.edu.

D01:10.1523/JNEUROSCI.5061-05.2006

Copyright $\odot 2006$ Society for Neuroscience $\quad$ 0270-6474/06/266485-11\$15.00/0 not known whether such neurons are sensitive to spatial features such as orientation. More generally, this raises a fundamental question about neurons with multidigit RFs: are they primarily processing information about local stimulus features that contact single digits, or are they integrating information about the features of large shapes that contact multiple digits? Finally, it is not known whether multidigit RF neurons in different cortical areas play different roles in processing cutaneous information (Whitsel et al., 1969; Burton et al., 1995; Krubitzer et al., 1995; Fitzgerald et al., 2004). Characterization of the RF structures of single-digit and multidigit neurons is a first step in understanding how tactile shape is represented in the somatosensory cortex.

In the current study, we sampled neurons throughout the SII hand region. We mapped the RF structures of the neurons with a motorized stimulator that pressed an oriented bar onto individual finger pads of the four large fingers (digits 2-5) of the hand. With this protocol, we address three principal questions about the RF properties of SII region hand neurons. First, we quantified the sizes and shapes and degree of excitation and inhibition of the RFs. Second, we determined which finger pads were orientation tuned. Third, we extend on our previous study (Fitzgerald et al., 2004) and quantify RF differences between the three SII region fields.

Parts of this work have been published previously in abstract form (Fitzgerald et al., 1999).

\section{Materials and Methods}

The details of the recording methods that were used in this study have been reported previously (Fitzgerald et al., 2004). Briefly, we recorded 
from single neurons in the SII hand region of four hemispheres of two rhesus monkeys (Macaca mulatta). Thirty-five to $45 \mathrm{~d}$ were spent recording in each hemisphere. Single-neuron data were collected while the monkeys performed a visual detection task that maintained them in a nearly constant state of alertness. The monkeys were trained to allow their hands to be restrained during the recording sessions, because this allowed for accurate and repeatable stimulation of the digits by the motorized tactile stimulator.

This stimulator, which was described by Fitzgerald et al. (2004), indented a small oriented bar (Altem plastic) onto individual finger pads of digits $2-5$ (of the hand contralateral to the recorded hemisphere) in a randomized sequence, generating eight repetitions at each of eight, $22.5^{\circ}$ separated bar orientations for the 12 finger pads of these four digits. Stimuli were presented in a pseudorandom order to minimize the time spent traveling between finger pads. After stimulating a pad with a random sequence of two repetitions of each of the eight orientations, the bar was moved to a randomly chosen neighboring finger pad. This sequence was repeated until each pad had been presented with eight repetitions of each of the eight orientations. Stimulus duration was $500 \mathrm{~ms}$, with an indentation force of $10 \mathrm{~g}$. The bar was approximately the width of a monkey's finger and had rounded ends; its short axis was a $90^{\circ}$ wedge, and its long axis was circular with an $8 \mathrm{~mm}$ radius, effectively producing a length of $\sim 7 \mathrm{~mm}$.

After the recordings were completed, each monkey was deeply anesthetized and perfused transcardially. Electrode tracks, which had been marked with fluorescent dyes (DiCarlo et al., 1996), were reconstructed in Neurolucida (MicroBrightField, Williston, VT) and AutoCAD (Autodesk, San Rafael, CA) to confirm that the recordings were made in the SII region.

Responsiveness of each neuron to the oriented bar stimuli was assessed in a two-step manner. In the first step, each of the 12 stimulated finger pads was tested for orientation tuning with a one-way ANOVA on the firing rates evoked during the $500 \mathrm{~ms}$ presentation of each of the eight, $22.5^{\circ}$ separated orientations (overall $p<0.05$, Bonferroni corrected for 12 comparisons). In the second step, each finger pad that did not exhibit tuning was tested for untuned excitatory or inhibitory responsiveness by pooling the data from all eight orientations and comparing the mean spontaneous firing rate ( $500 \mathrm{~ms}$ window before stimulus onset) with the mean rate evoked during the $500 \mathrm{~ms}$ presentation of the oriented bars (unpaired two-tailed $t$ test, overall $p<0.05$, Bonferroni corrected for up to 12 comparisons). Thus, in this two-step manner, each finger pad was classified as orientation tuned, untuned excitatory, untuned inhibitory, or not responsive, and five mutually exclusive RF types (see Figs. 1-9) were created based on combinations of these four pad types comprising the 12 stimulated finger pads of a given neuron. A neuron with at least one finger pad that was orientation tuned, untuned excitatory, or untuned inhibitory was considered responsive to the oriented bar stimuli. Similarly, for a given neuron, a digit was considered responsive if it contained one or more responsive pads. The preferred orientation of each tuned pad was calculated as the mean angular vector of the eight presented orientations, in which the magnitude of each of these eight individual vectors (used to calculate the mean angular vector) was equal to the mean firing rate at its given orientation.

For the RF diagrams shown in Figures 2, 5, 7, and 8, the degree of excitation (redness) or inhibition (blueness) of each pad is normalized to the maximum deviation from the spontaneous firing rate of the $96 \mathrm{pad} /$ orientation stimulus combinations $(12$ pads $\times 8$ orientations $=96 \mathrm{com}$ binations) for that neuron. In addition, to calculate the degree of redness or blueness, we used the inverse of a sigmoid function to compress the variability of coloration intensity for pads that had evoked firing rates near 0 (spontaneous firing rate) and expand the variability between pads with greater deviations in evoked firing rate.

The analyses shown in Figures 11 and 13, as well as the analysis of the relative location of the two most responsive pads, required rank ordering finger pads based on responsiveness. When there were ties in the ranks that confounded the results, we excluded those neurons from the particular analysis.

We classified each neuron as belonging to the SII region posterior (p), central (c), or anterior (a) field based on the subjective multiunit responses to hand-held stimuli (for details, see Fitzgerald et al., 2004).

\section{Results}

We studied 928 SII region neurons with RFs on the hand, the same neurons studied by Fitzgerald et al. (2004). A total of 749 ( $81 \%)$ of these neurons were responsive to the oriented bar stimuli (see Materials and Methods), and most of these responsive neurons had RFs that spanned multiple digits (605 of 749, 81\%). What follows is a description of the RF characteristics of the neurons and a comparison of these characteristics between the SII region posterior, central, and anterior fields. First, we describe the different RF types that we encountered, and then we quantify the RF sizes, shapes, and firing rates. Finally, we discuss the somatotopic representation of the hand in the neural responses.

\section{Receptive field type}

Based on the responses to the motorized stimulator, we used five mutually exclusive RF types (see Figs. 1-9) to classify posterior field $(n=146)$, central field $(n=372)$, and anterior field $(n=$ 410 ) neurons. These RF types were based on the response properties of the finger pads that comprised the neuronal RFs and included three broad classes of neurons: those with only untuned pads that had excitatory or inhibitory responses (types UE, UI, and UEI), those with one or more tuned pads (type T), and those that were not responsive to the stimuli (type NR).

Most of the neurons had only untuned RFs, with 36\% having only untuned excitatory pads [type UE (see Figs. 1-3)], 17\% having only untuned inhibitory pads [type UI (see Figs. 3-5)], and $5 \%$ having both untuned excitatory and untuned inhibitory pads [type UEI (see Figs. 3, 6, 7)]. The second largest group (23\%) consisted of neurons that had at least one orientation tuned pad [type T (see Figs. 3, 8)], and most of these neurons had additional untuned pads. Finally, 19\% of the neurons were not responsive (type NR) to the oriented bar stimuli.

\section{Type UE neurons}

The most common type of neuron in the SII region was the untuned excitatory type UE. Figure 1 shows raster plots and peristimulus time histograms (PSTHs) for an example type UE central field neuron. This neuron had the following untuned excitatory pads: D2m, D2p, D3m, D3p, D4m, D4p, D5d, and D5p. Its firing rate was highest when D2p, D3m, and D3p were stimulated. It tended to give sustained responses to the indented oriented bar, and there were no significant differences in firing rate between orientations for its responsive pads.

Figure 2 shows RF diagrams for a random sampling of 45 type UE neurons. Type UE neurons in each SII region field exhibited a range of RF sizes, from a single pad to multiple pads on multiple digits. In addition, the RFs varied greatly in shape, from a simple homogeneous cluster of pads (such as neurons d4 and g5 in Fig. 2) to complex RFs with noncontiguous clusters (such as neuron h4 in Fig. 2). Typically, UE neurons with multidigit RFs had different structures on separate digits, and peak firing rate was not uniform across the responsive pads. Within the $3 \times 4$ grid of stimulated pads, most RFs show spatial gradients in firing rate (such as neurons i2 and b5 in Fig. 2), whereas others do not, particularly those whose pads are not contiguous (such as neurons a 4 and h4 in Fig. 2).

Figure 3 shows the distribution of RF sizes for all four types of responsive neurons, measured as the number of responsive finger pads. The mean number of untuned excitatory pads for type UE neurons was as follows: p field, 5.82; c field, 6.42; a 


\section{Type UE Neuron}

CL01A_14

D2
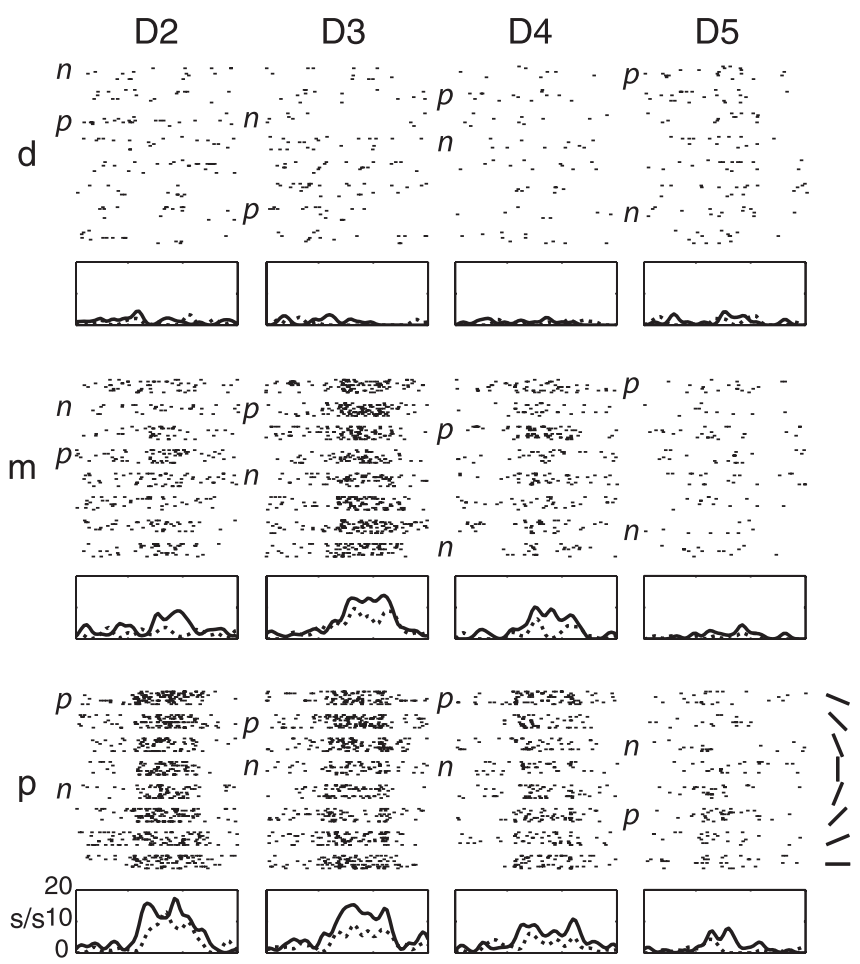

$1 \mathrm{sec}$

Figure 1. Type UE neuron raster and PSTH. Type UE neurons had only untuned excitatory finger pads. In the raster, stimulus trials are first sorted into the 12 stimulated finger pads (D2-D5, d-p) and then within each pad are sorted by the orientation of the bar (see bottom right). The bar stimulus indentation profile is also shown (bottom right). Each PSTH graph is shown below its corresponding finger pad, in which only the preferred (highest firing rate; solid line) and nonpreferred (lowest firing rate; dashed line) orientation rates are plotted after convolving these rates with a Gaussian (raw bin size before smoothing was $25 \mathrm{~ms}$; Gaussian $\sigma$ is $35.4 \mathrm{~ms}$ ). Shown is central field neuron CL01A_14. D, Digit; $d$, distal; $m$, middle; $p$, proximal; $p$, preferred orientation; $n$, nonpreferred orientation; $s / s$, spikes per second.

field, 5.66; overall, 5.99. All possible sizes, from 1 to 12 pads, are represented in each of the three fields. The $\mathrm{p}$ field exhibits a peak in the number of neurons with one-pad RFs, and the a field distribution is $U$-shaped, with a peak in the number of neurons with small and large RFs. The overall distribution is also U-shaped.

\section{Type UI neurons}

A less common type of neuron in the SII region was the untuned inhibitory type UI. Figure 4 shows raster plots and PSTHs for a typical type UI neuron, which in this case was from the posterior field. All 12 of the pads of this neuron were untuned inhibitory, and its firing rate was fairly similar across pads. It tended to give transient responses (with some additional sustained inhibition) to the oriented bar, becoming most inhibited during indentation and withdrawal of the bar, with slight and insignificant differences in firing rate evoked by the different bar orientations for each pad.

Figure 5 shows RF diagrams for a random sampling of 45 type UI neurons. Like the type UE neurons, type UI neurons in each field exhibited a range of sizes (from small to large) and shapes (from simple to complex). Moreover, many of these neurons had

\section{Type UE RF Diagrams}

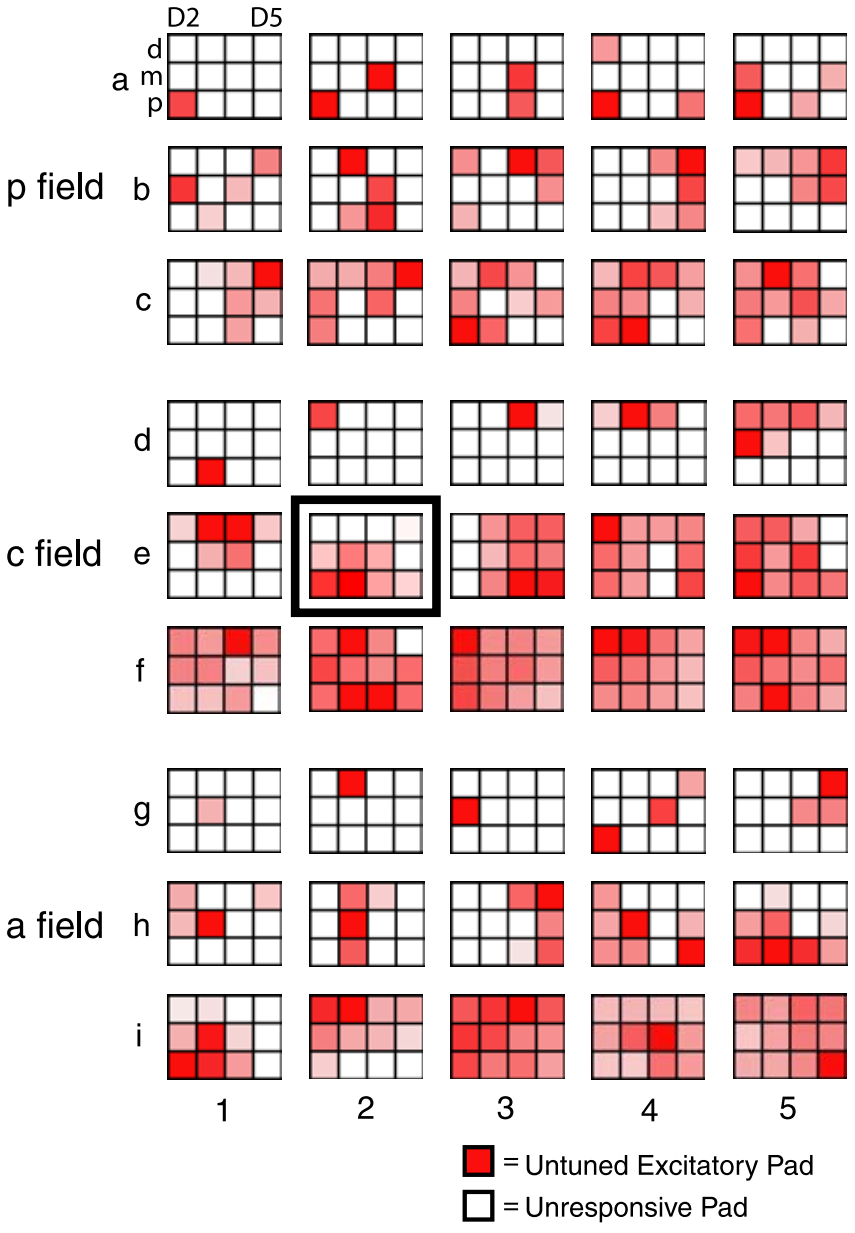

Figure 2. Type UE receptive fields. Shown are receptive field diagrams of a random sampling of 15 posterior field, 15 central field, and 15 anterior field type UE neurons. $\boldsymbol{a}$ - $\boldsymbol{c}$ represent posterior field neurons, $\boldsymbol{d}-\boldsymbol{f}$ represent central field neurons, and $\boldsymbol{g}$-i represent anterior field neurons. Each $3 \times 4$ grid represents the 12 pads of D2-D5 for a single neuron (as shown for the top left receptive field diagram), with the top row of each grid representing the distal pads and the left column representing D2 (in which left-hand and right-hand receptive fields are interspersed, and right-hand receptive fields are reflected leftward). Each white square is an unresponsive pad, whereas each red square is an untuned excitatory pad. The degree of redness of each untuned excitatory pad is normalized to the maximum deviation from the spontaneous firing rate of the $96 \mathrm{pad} /$ orientation stimulus combinations and represents the peak excitatory rate (preferred orientation) of each untuned excitatory pad. Within each SII region field, the neurons are arranged in ascending order based on RF size (number of pads). The receptive field diagram for type UE neuron CL01A_14 (Fig. 1) is framed and shown in position e2.

different RF structures on different digits, and many showed differences in peak firing rate across pads. Within the $3 \times 4$ grid of stimulated pads, some RFs show spatial gradients in firing rate (such as neurons $\mathrm{f} 1$ and $\mathrm{f} 2$ in Fig. 5), whereas others do not, particularly those whose pads are not contiguous (such as neurons h2 and b5 in Fig. 5).

Figure 3 shows the distribution of RF sizes for type UI neurons, in which the mean numbers of pads were as follows: $p$ field, 3.24; c field, 4.65; a field, 4.02; overall, 4.12, which are smaller than for the UE neurons. Each of the three fields exhibits a peak at small sizes (one or two pads), and the $c$ and a fields exhibit a peak at 12 pads as well. The overall distribution contains all possible sizes (1-12 pads) and is U-shaped, with a large peak at small sizes and a small peak at large sizes. 

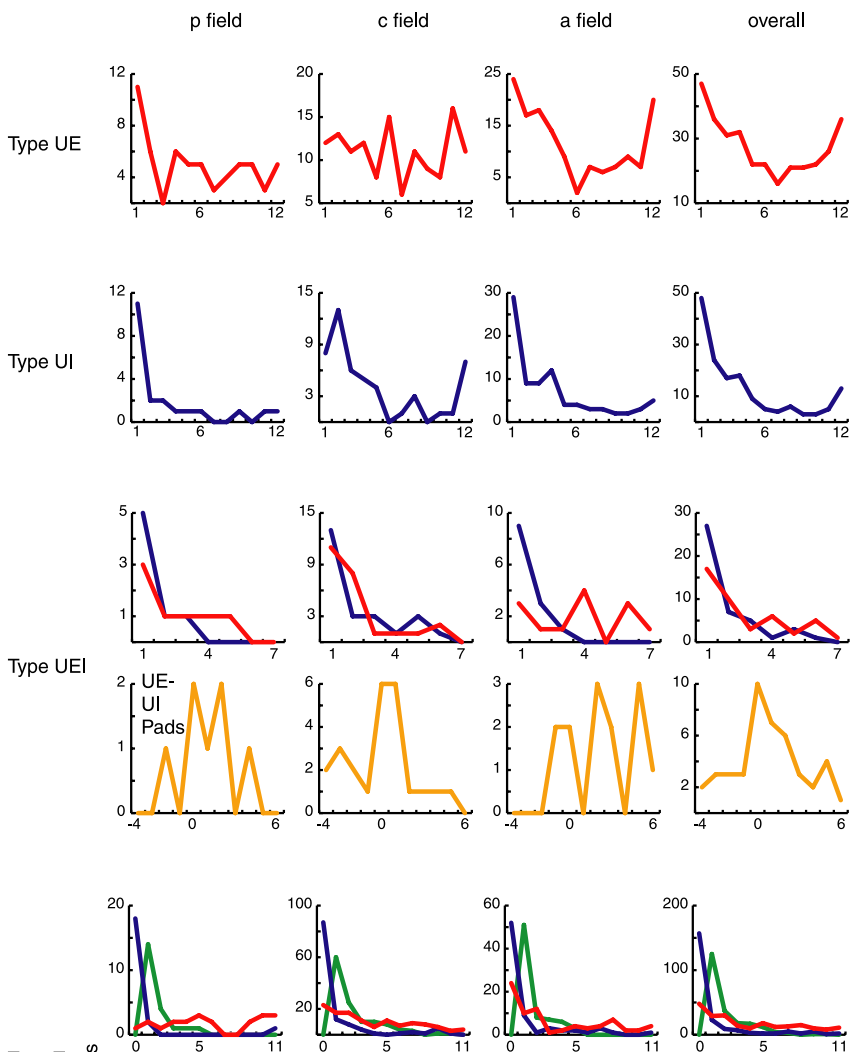

Type T
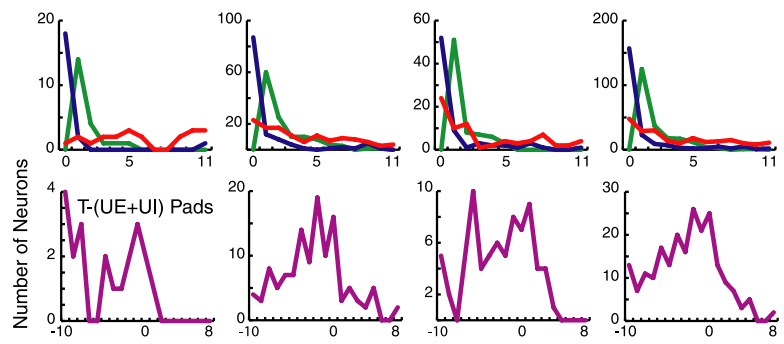

$$
\begin{aligned}
& \text { UE Pads } \\
& \text { - UI Pads } \\
& \text { T Pads }
\end{aligned}
$$

Figure 3. Receptive field size. Shown are receptive field sizes based on the number of neurons with the given number of responsive finger pads for each of the four responsive neuron types (UE, UI, UEI, and T). For type UE neurons, the number of UE pads (red) per neuron is shown. For type UI neurons, the number of UI pads (blue) is shown. For type UEl neurons, the number of UE and UI pads are shown independently, as well as the number of UE pads minus the number of UI pads (orange). For type T neurons, the number of UE, UI, and T pads (green) are shown independently, as well as the number of T pads minus the sum of UE and UI pads (purple). The overall distribution represents the total number of neurons of the given type from all three fields.

\section{Type UEI neurons}

A rare type of neuron in the SII region was the untuned excitatory/inhibitory type UEI. Figure 6 shows raster plots and PSTHs for an example type UEI central field neuron that had two untuned excitatory pads (D3d and D4d) and five untuned inhibitory pads (D2p, D3p, D4p, D5p, and D5m). It tended to give sustained responses to the oriented bar, with the firing rate on the untuned excitatory pads ramping upward during indentation and after the bar left the skin. For most type UEI neurons, such as the one shown in Figure 6, there was a gradual transition from excitation in one region of the RF to inhibition in another region, with pads at intermediate locations showing intermediate properties.

Figure 7 shows RF diagrams for 35 type UEI neurons. Like the other untuned neurons, the type UEI neurons in each field exhibited a range of sizes (from small to large) and shapes (from simple to complex) and tended to have different RF structures on different digits. These neurons also tended to have a peak firing rate
CMOOC 8

\section{Type UI Neuron}

D2

\author{
D3
}

D4

D5
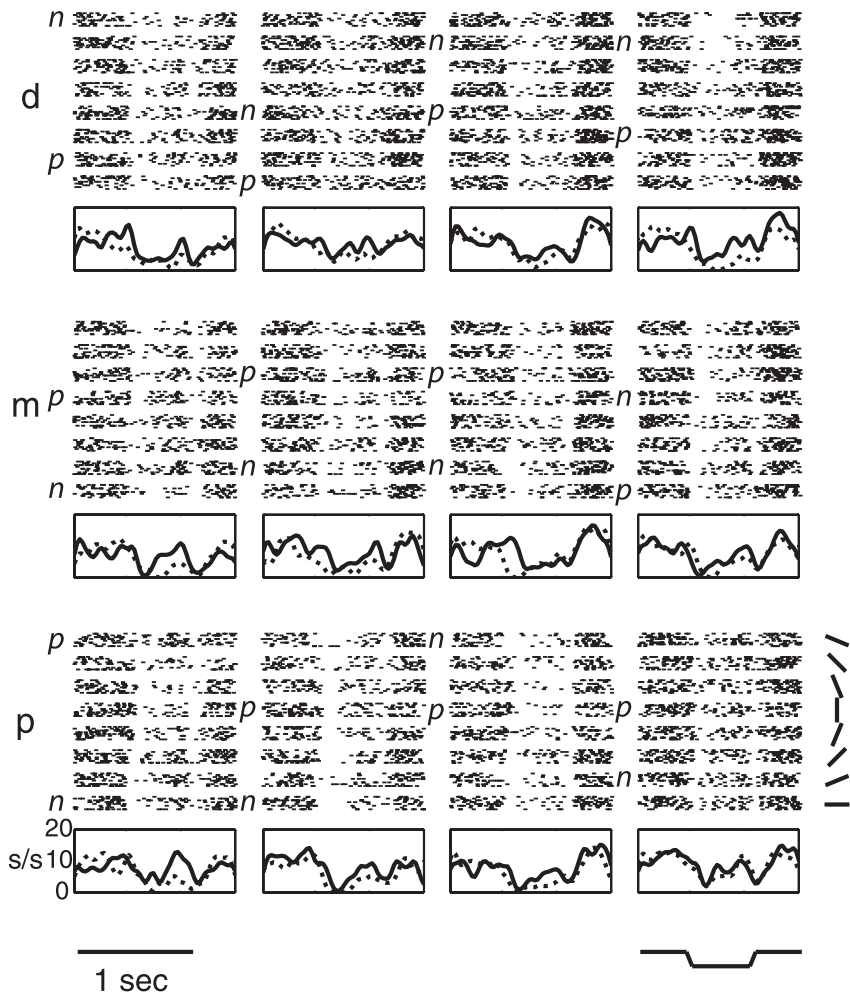

Figure 4. Type UI neuron raster and PSTH. Type UI neurons had only untuned inhibitory finger pads. Raster and PSTH graphs are arranged as in Figure 1, with the same abbreviations. Shown is posterior field neuron CMOOC_8.

that was not uniform within the RF, even within just the excitatory pads or the inhibitory pads of a single neuron. In addition, for many of the neurons, the peak magnitude of excitation tended to be equal to or greater than the peak magnitude of inhibition. Finally, for most type UEI RFs, the excitatory and inhibitory pads are not randomly distributed throughout the hand but instead have separate lobes (made up of one or more contiguous pads) of excitation and inhibition.

Figure 3 shows the distribution of RF sizes for type UEI neurons, showing the number of UE and UI pads for each neuron independently (top row of graphs) and also showing total UE pads minus total UI pads for each neuron [bottom row of graphs; mean number of pads (UE - UI): p field, 1.00; c field, -0.09 ; a field, 2.39; overall, 0.82]. For the independent measures of UE and UI pads, all of the distributions have a peak at small RF sizes and decrease rapidly toward larger sizes, and the UI distributions decrease more rapidly than the UE distributions. For the UE minus UI graphs, the $\mathrm{p}$ and a fields, as well as the overall distribution, have the majority of neurons falling to the right of 0 , illustrating that type UEI neurons tend to show greater ratios of excitatory-to-inhibitory pad counts.

\section{Type T neurons}

The fourth and perhaps most interesting type of responsive neuron had one or more orientation tuned pads (type T). Figure 8 shows RF diagrams for a random sampling of 45 type T neurons. In each SII region field, type $\mathrm{T}$ neurons exhibited a range of sizes and shapes, and, in general, these neurons had different RF struc- 


\section{Type UI RF Diagrams}
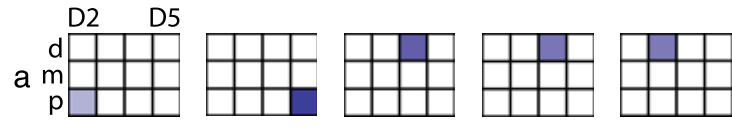

p field
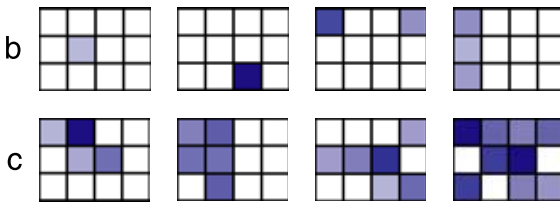

C

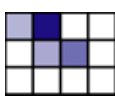

d
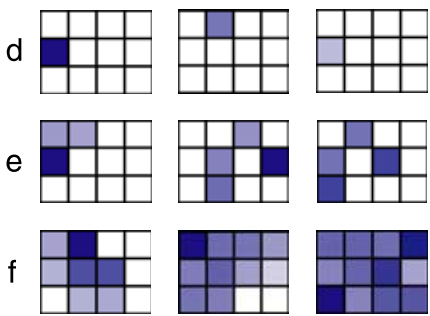

f

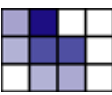

g
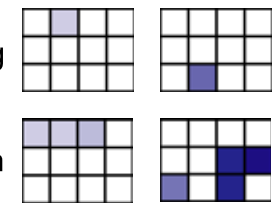

i

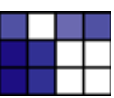

1

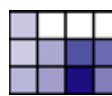

2
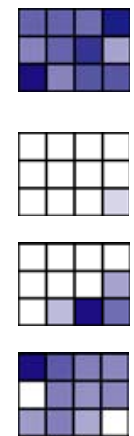

3
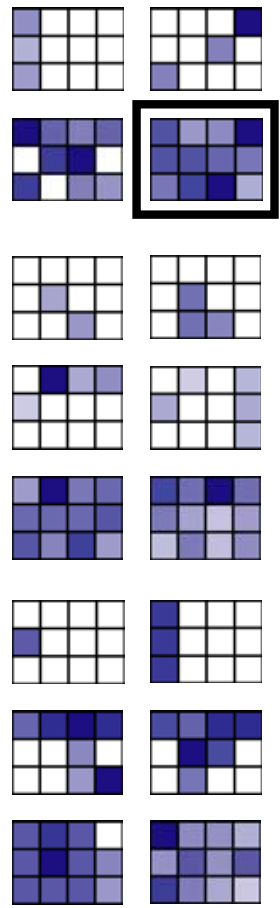

4

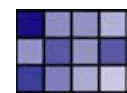

5

$\square=$ Untuned Inhibitory Pad

$\square=$ Unresponsive Pad

Figure 5. Type UI receptive fields. For more details, see the legend of Figure 2. Each blue square is an untuned inhibitory pad. The degree of blueness of each untuned inhibitory pad is normalized to the maximum deviation from the spontaneous firing rate of the $96 \mathrm{pad} /$ orientation stimulus combinations and represents the peak inhibitory rate (nonpreferred orientation) of each untuned inhibitory pad. The receptive field diagram for type UI neuron CMO0C_8 (Fig.4) is framed and shown in position $\mathrm{c5}$.

tures on separate digits. Type T neurons also tended to have peak firing rates that were not uniform across pads, either within or across each of their up to three types of responsive pads (tuned, untuned excitatory, and untuned inhibitory).

Most type $\mathrm{T}$ neurons had additional untuned excitatory and/or untuned inhibitory pads (Fig. 8). Type T neurons with only tuned pads were very rare in all three SII region fields and only accounted for a total of 12 neurons (such as neurons $\mathrm{g} 1$ and g4 in Fig. 8). These 12 neurons were found only in the central $(n=2)$ and anterior $(n=10)$ fields, and, for most ( 8 of 12$)$ of the neurons, the RF consisted of only a single tuned pad. For most type $\mathrm{T}$ neurons with multiple tuned pads, the tuned pads tended to cluster together (such as neuron d4 in Fig. 8), but this was not always the case (such as neuron i3 in Fig. 8).

Figure 3 shows the distribution of RF sizes for type T neurons, showing the number of UE, UI, and $\mathrm{T}$ pads for each neuron independently (top row of graphs) and also showing the number of tuned pads minus the sum of all untuned pads of each neuron (bottom row of graphs; mean number of pads $[\mathrm{T}-(\mathrm{UE}+\mathrm{UI})]$ : p field, -5.00 ; c field, -2.48 ; a field, -2.75 ; overall, -2.82 ), which demonstrates that these neurons on average had a greater number of untuned than tuned pads. For the independent mea-

\section{Type UEI Neuron}

CJ03H_10
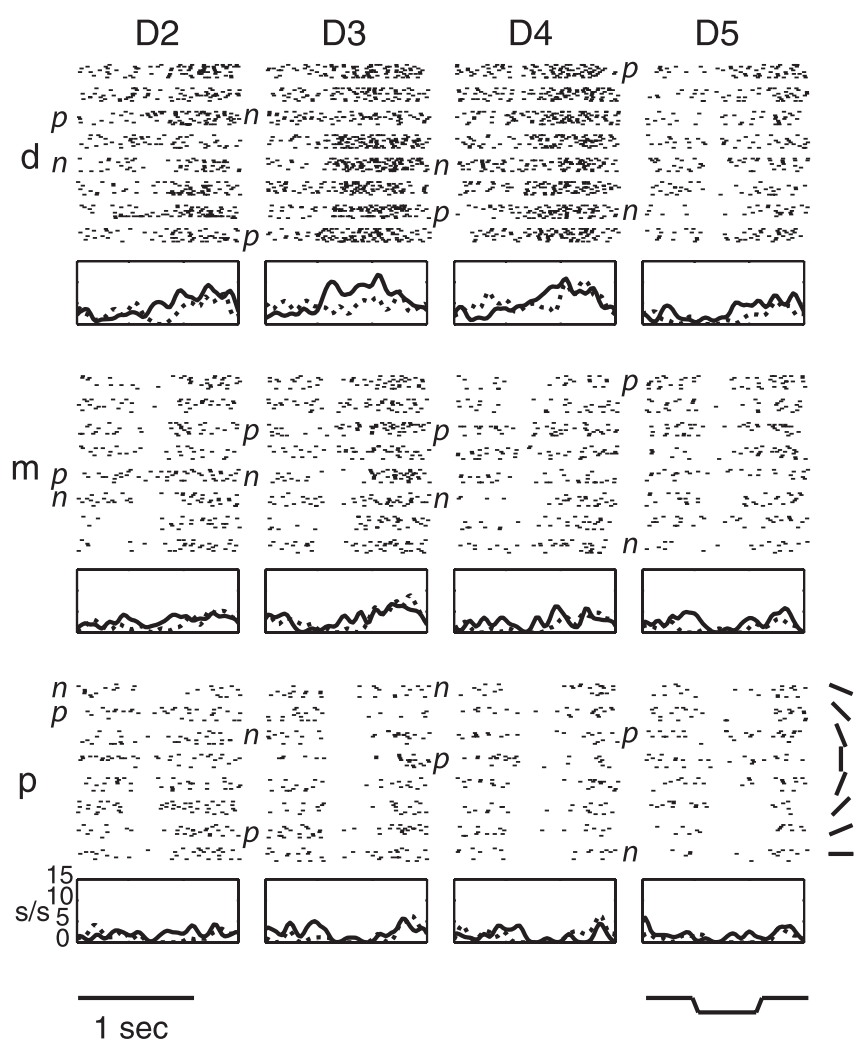

Figure 6. Type UEl neuron raster and PSTH. Type UEl neurons had both untuned excitatory and untuned inhibitory finger pads. Raster and PSTH graphs are arranged as in Figure 1, with the same abbreviations. Shown is central field type UEl neuron CJ03H_10.

sures of UE, UI, and T pads, most of the distributions have a peak at small RF sizes and decrease toward larger sizes, in which the UI distributions decrease most rapidly and the UE distributions decrease least rapidly. For the tuned minus untuned graphs, the $c$ and a fields, as well as the overall distribution, exhibit inverted U-shaped distributions.

\section{Distribution of receptive field types}

Figure 9 shows the percentages of the five RF types in each of the three SII region fields. In each of the three fields, the exclusively untuned excitatory (type UE) neurons were the most numerous, and the type UEI neurons were the least numerous. The percentages of posterior and anterior field neurons were similar for all neuron types but differed from central neurons, which showed a relative increase in tuned (type T) neurons and decrease in not responsive (type NR) neurons. These results suggest that the central field is specialized for processing information about the orientation of features on the skin, consistent with the hypothesis that these are three separate fields that play different functional roles in tactile perception.

\section{Additional measures of receptive field size and shape}

As can be seen in Figures 1-9, RF size varied greatly across neurons in each of the three SII region fields. To further quantify this, we collapsed the data across all neuron types and examined the number of responsive finger pads and number of responsive digits for all 928 neurons, in which a digit was considered responsive if it contained one or more responsive pads. All possible sizes 


\section{Type UEI RF Diagrams}

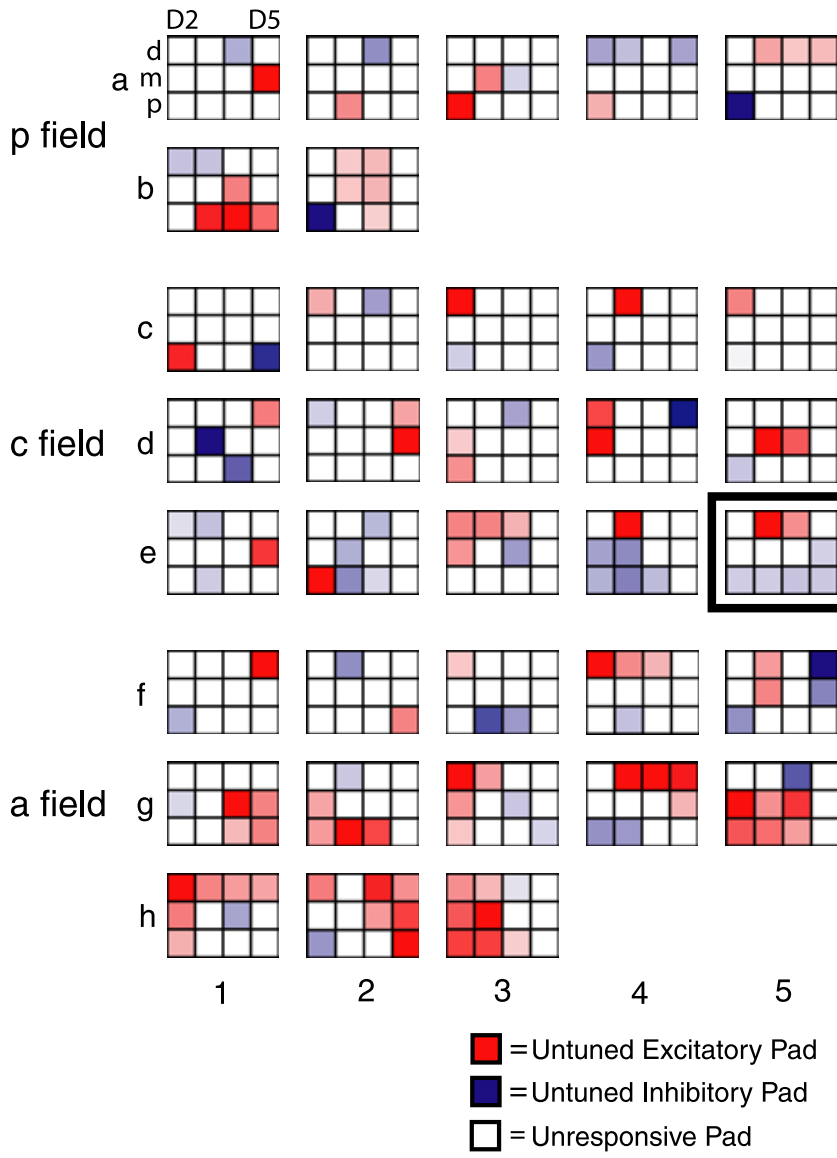

Figure 7. Type UEl receptive fields. Shown are receptive field diagrams of 35 type UEI neurons, including all seven such posterior field neurons, a random sampling of 15 such central field neurons, and all 13 such anterior field neurons. $\boldsymbol{a}$ and $\boldsymbol{b}$ represent posterior field neurons, $\boldsymbol{c}-\boldsymbol{e}$ represent central field neurons, and $\boldsymbol{f}$ - $\boldsymbol{h}$ represent anterior field neurons. For more details, see Figure 2. The receptive field diagram for type UEI neuron CJ03H_10 (Fig.6) is framed and shown in position e5.

(0-12 pads; $0-4$ digits) were represented in each of the three SII region fields. Mean RF size for responsive neurons was largest in the central field and smallest in the anterior field, both for number of pads [posterior field, 5.66; central field, 6.27; anterior field, 5.31; one-way ANOVA, $p<0.005$; central field $>$ anterior field, Tukey's honestly significant difference (HSD) post hoc test, $p<$ 0.005 ] and number of digits (posterior field, 2.81; central field, 3.08; anterior field, 2.67; one-way ANOVA, $p<0.001$; central field $>$ anterior field, Tukey's HSD post hoc test, $p<0.001)$. For each of the three fields, the data are not uniformly distributed across all RF sizes, because there was an overrepresentation of neurons with small and large RF sizes.

The spread of the RFs across digits for all neuron types is shown in Figure 10, which depicts the total number of responsive pads versus digits, per neuron. Figure $10 \mathrm{~A}$ shows that there is a tendency for neurons with one- to three-digit RFs to have a minimal number of responsive pads (one responsive pad per digit), whereas neurons with four-digit RFs tend to have a maximal number of responsive pads (three responsive pads per digit). The abundance of large RFs observed with four-digit RFs may exist because these may include neurons with RFs covering larger portions of the hand than we were able to study with our stimulator. Figure $10 B$ summarizes these data and shows that there was a

\section{Type T RF Diagrams}

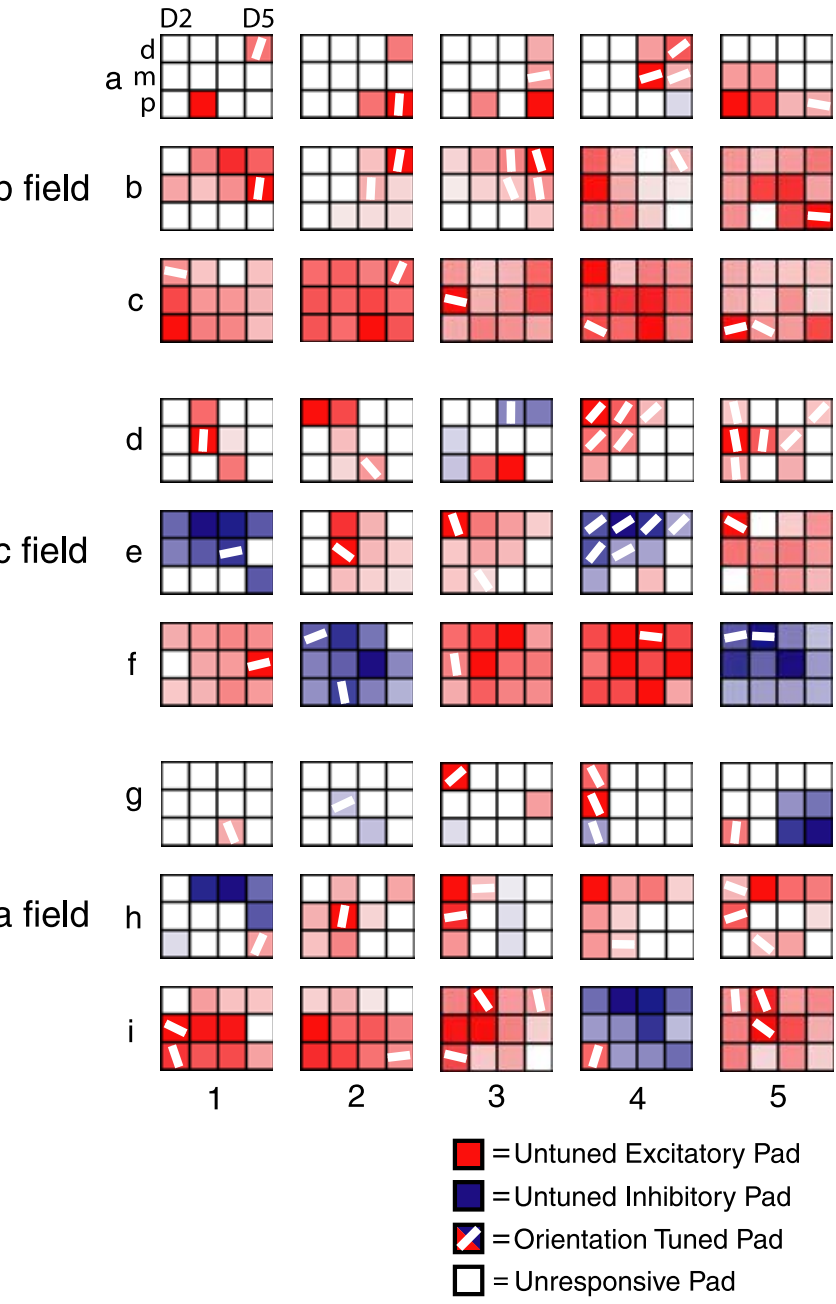

Figure 8. Type T receptive fields. Type T neurons had one or more orientation tuned finger pads, and most had additional untuned pads. For more details, see Figure 2. Each red or blue square with a superimposed white bar is an orientation tuned pad. The degree of redness or blueness of each colored pad is normalized to the maximum deviation from the spontaneous firing rate of the $96 \mathrm{pad} /$ orientation stimulus combinations. For untuned excitatory pads, redness represents the peak excitatory rate (preferred orientation); for untuned inhibitory pads, blueness represents the peak inhibitory rate (nonpreferred orientation); and for tuned pads, redness or blueness represents the maximum deviation from the spontaneous rate. The orientation of each white bar represents the preferred orientation (mean angular vector) of that tuned pad. As in Figures 2, 5, and 7, both right-hand and left-hand receptive fields are plotted with D2 in the left column of each $3 \times 4$ grid, although the preferred orientations of tuned pads are not reflected.

tendency toward maximal spreading of the RF across separate digits at lower numbers of digits and minimal spreading at higher numbers of digits. The results in Figure 10 are similar for the three SII region fields and suggest that RFs in the SII region spread more strongly across separate digits than along the same digit, consistent with the hypothesis that neurons in the SII region are involved in integrating information across digits.

In Figure 11, we address the relationship between RF size and firing rate. This figure shows how rapidly the peak firing rate of each responsive pad decreased when the pads are rank ordered from most responsive (highest peak rate; left) to least responsive (lowest peak rate; right), in which each curve represents the average response across neurons with $n=1-12$ responsive pads in the given SII region field. These graphs illustrate three points. 


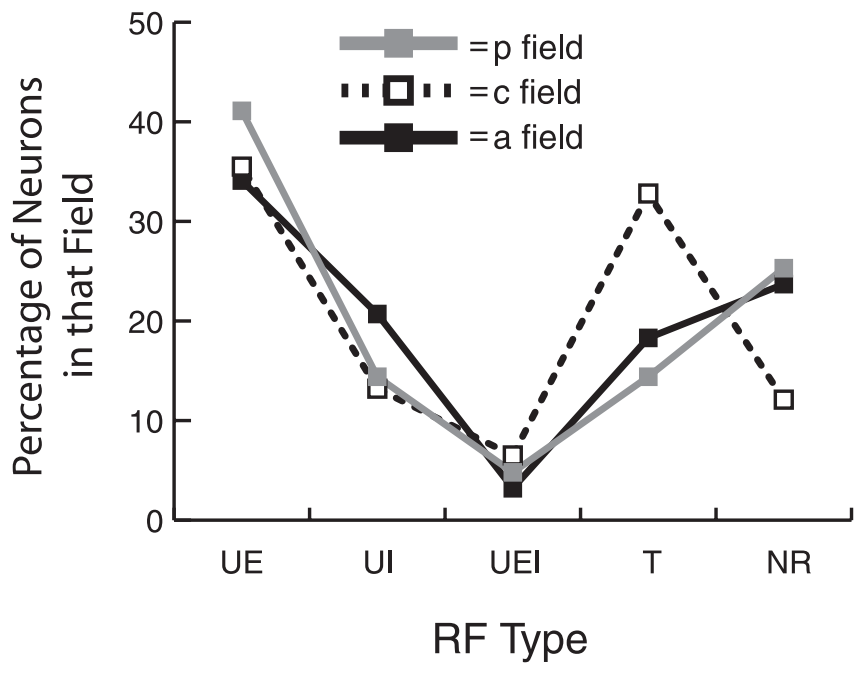

Figure 9. Receptive field types. Shown are the percentages of the five receptive field types (UE, UI, UEl, T, and NR) within each field.

First, although on average, the pads are not equally responsive across the RF, the decrease in firing rate across pads tends to be fairly mild, typically showing a ratio of $<3$ between most responsive pad and least responsive pad. Second, neurons with large RFs tend to have higher firing rates than neurons with small RFs, especially in the central and anterior fields. Third, the firing rates tend to be highest in the central field and lowest in the posterior field, although the differences are small.

We addressed whether the relative locations of the responsive pads are randomly distributed among the four digits by analyzing the positions of the two most responsive (highest peak firing rate) pads, determining whether they were on the same digit, adjacent digits, or nonadjacent digits. If the two most responsive pads are randomly distributed among the four stimulated digits, then the chance values are as follows: 2 of $11=0.18$ for same digit; 4.5 of $11=0.41$ for adjacent digits; and 4.5 of $11=0.41$ for nonadjacent digits. The observed proportions in the three SII region fields are well above chance (posterior field, 0.30; central field, 0.29; anterior field, 0.33) for same digit, closer to chance (posterior field, 0.36; central field, 0.50; anterior field, 0.43) for adjacent digits, and well below chance (posterior field, 0.34; central field, 0.21; anterior field, 0.24) for nonadjacent digits. So at least for the central and anterior fields, the two most responsive pads tend to cluster within the same digit or on adjacent digits, suggesting that many neurons in the SII region serve an integrative function for stimuli contacting adjacent pads.

To further address how the responsive pads are distributed within and across separate digits, we next asked whether the RFs of neurons with multiple responsive pads were contiguous. An $\mathrm{RF}$ is defined as contiguous if all responsive pads are interconnected (within the $3 \times 4$ grid of stimulated pads) such that no pad or group of pads is separated from the remaining group or groups by spatially intervening unresponsive pads. Chance was calculated with separate simulations based on a random distribution of $n=2-12$ responsive pads throughout the 12 stimulated pads. For most values of $n$ responsive pads, a greater proportion of neurons were contiguous than expected by chance, indicating that the pads in these RFs are not distributed randomly within the hand (Fig. 12). Nonetheless, as inspection of Figures 2, 5, 7, and 8 illustrates, a substantial proportion of the RFs were not contiguous. The results were similar for the central and anterior field
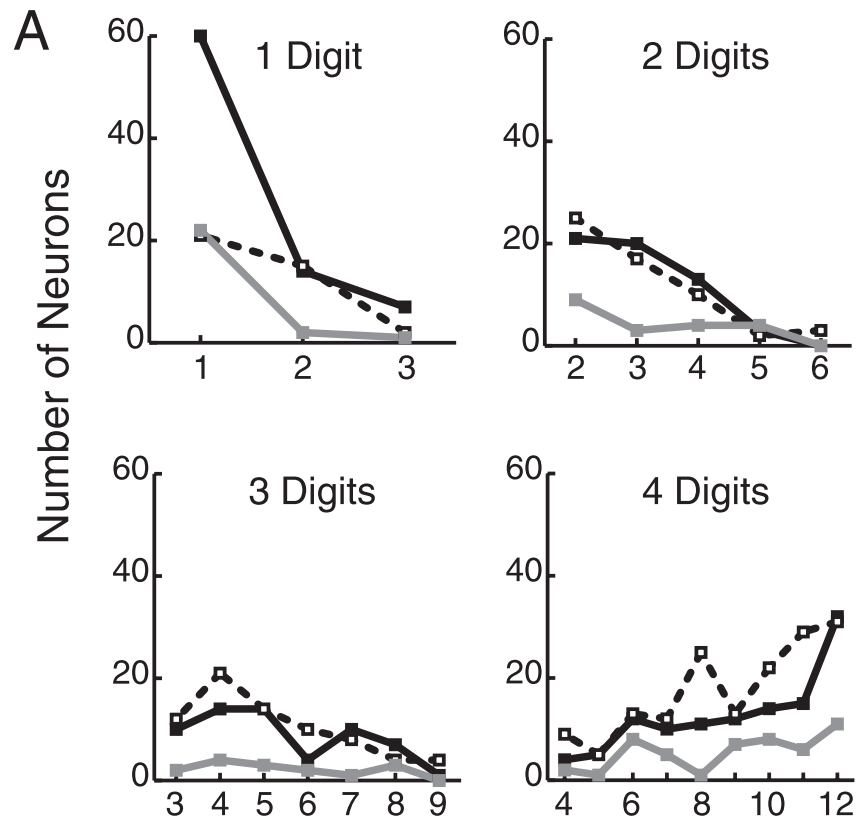

Number of Pads

B

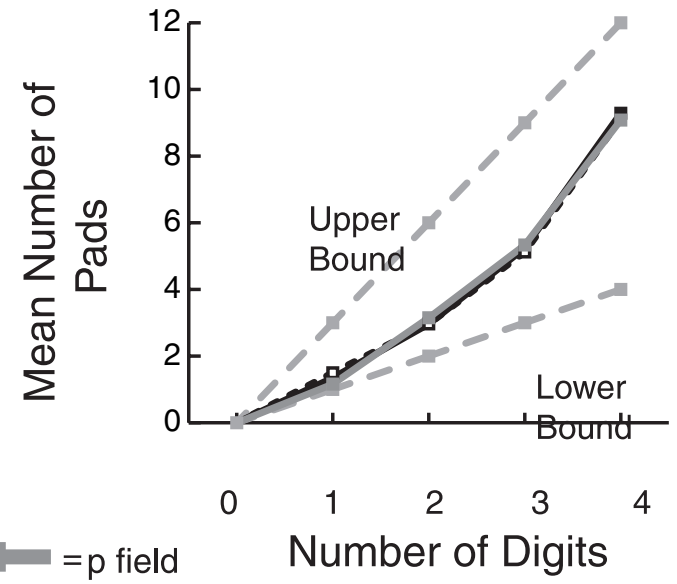

I I I I = c field

= a field

Figure 10. Number of responsive pads versus digits, per neuron. $A$, Number of responsive pads per responsive digit for each neuron. A pad was defined as responsive if it was untuned excitatory, untuned inhibitory, or orientation tuned (see Materials and Methods). A digit was defined as responsive if it contained one or more responsive pads. So every one-digit neuron could have one to three responsive pads, every two-digit neuron could have two to six responsive pads, every three-digit neuron could have three to nine responsive pads, and every fourdigit neuron could have $4-12$ responsive pads. $B$, Mean number of responsive pads per responsive digit for each neuron. The upper and lower bounds indicate the maximum and minimum number of pads per digit, respectively.

neurons, with the posterior field neurons showing less contiguity than the other two fields at small RF sizes.

\section{Somatotopy}

We also investigated whether SII region neurons are processing information from all 12 finger pads equally or whether there is a bias in how the digits are represented. The results, shown in Figure 13 , suggest that there is a bias in the anterior and central fields toward processing inputs from the lateral digits (D2 and D3) 

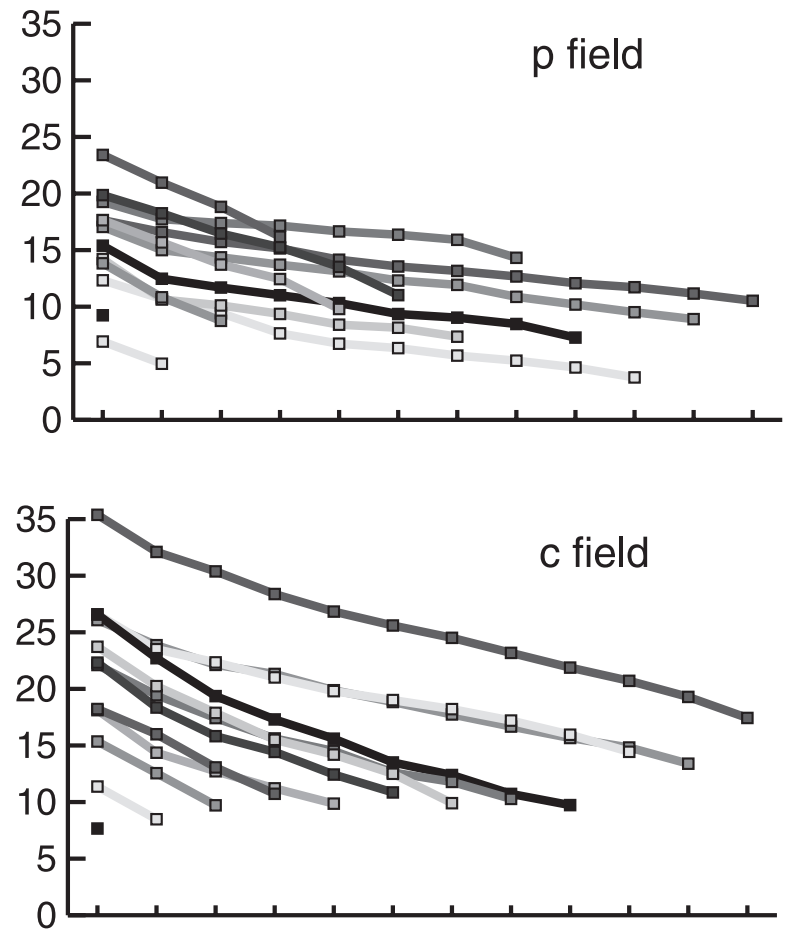

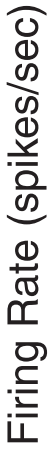

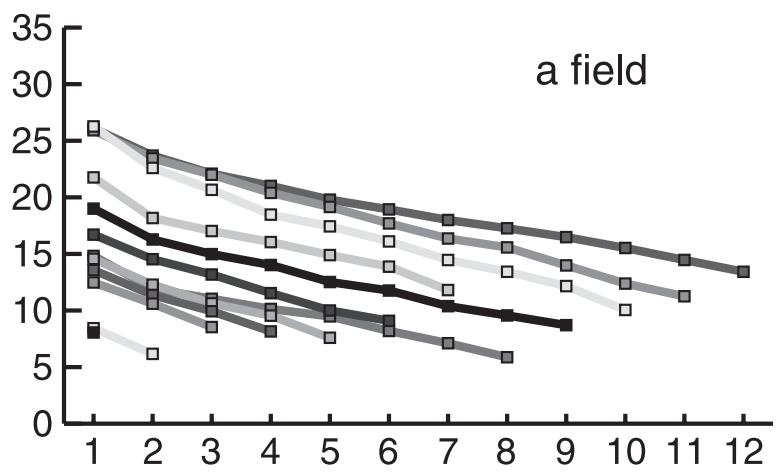

Pad Rank

Figure 11. Falloff in responsiveness. Each curve represents the mean decrease in peak firing rate (preferred orientation rate) across pads for neurons with $n$ responsive pads (in which rate is averaged across all neurons with the given number of responsive pads in the given SII region field). $n$ is between 1 and 12 , and pads are rank ordered from highest firing rate (left) to lowest firing rate (right).

rather than from the medial digits (D4 and D5) and that there is a bias in these two fields for processing inputs from distal rather than middle or proximal finger pads. These biases appear to be more pronounced for the best (highest firing rate) pad in the RF (B) than for all pads in the RF $(A)$. These results show that the finger pads that are most commonly used in manipulating and exploring objects (D2d and D3d) may be better represented in the SII region than other finger pads.

\section{Discussion}

General receptive field properties and possible functional roles of the neurons

A primary aim of this study was to characterize the RF structures of neurons in the SII region using controlled stimuli. Here we used a motorized, computer-controlled stimulator that delivered oriented bar stimuli to the immobilized hand to produce accurate

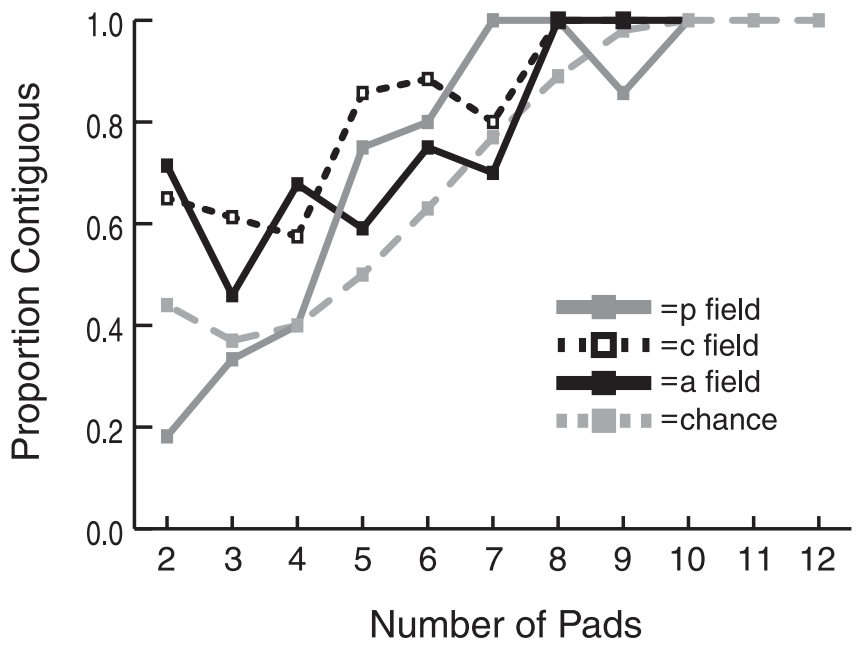

Figure 12. Contiguity. A receptive field is defined as contiguous if all of its responsive pads are interconnected (within the $3 \times 4$ grid of stimulated pads) such that no pad or group of pads is separated from the remaining group or groups by spatially intervening unresponsive pads. Therefore, contiguity is only relevant for neurons with two or more responsive pads. Diagonally bordering pads are considered contiguous. Chance (gray dashed line) was calculated with separate simulations based on a random distribution of $n=2-12$ responsive pads throughout the 12 stimulated pads.

A

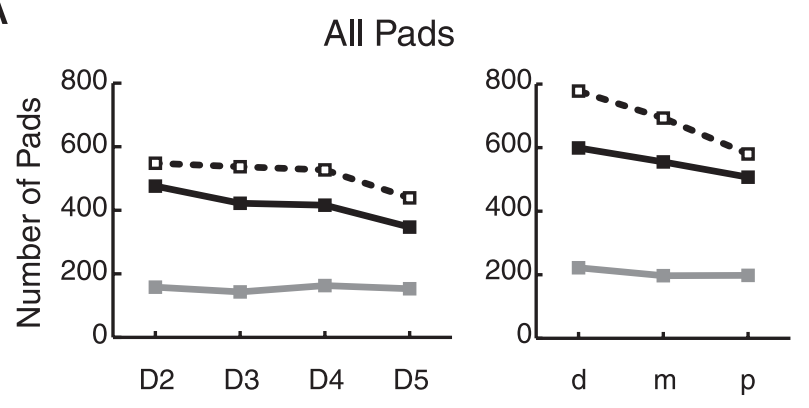

B

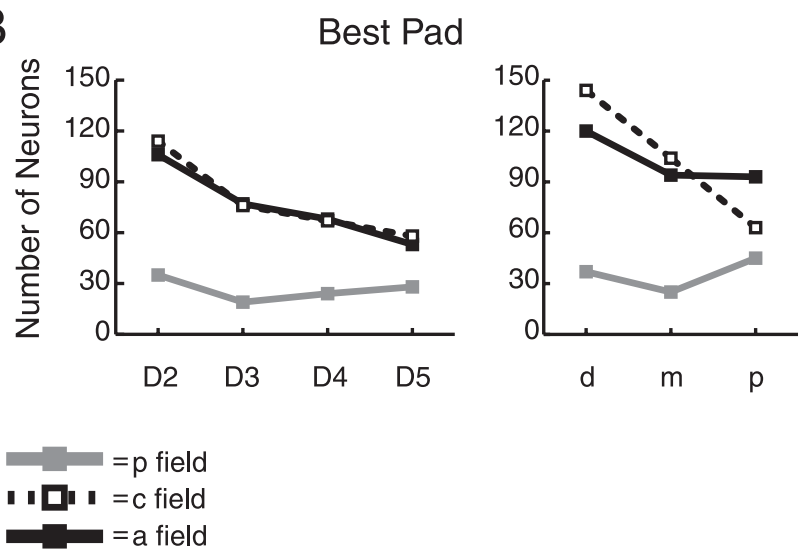

Figure 13. Somatotopic representation of the digits. $A$, Representation of digits and pads for all responsive pads. $\boldsymbol{B}$, Representation of digits and pads for the best pad of each responsive neuron. Best pad is defined as the responsive pad with highest firing rate at its preferred orientation.

RF maps of the cutaneous responses of a large number of neurons $(n=928)$ and thereby provided an accurate representation of cutaneous responses across all three SII region fields. We demonstrate that most SII region hand neurons have RF sizes that range 
from a single finger pad to those that span multiple finger pads and digits. The RFs also vary widely in complexity, from simple RFs composed of a few adjacent pads with similar response properties to complex RFs composed of combinations of tuned, untuned excitatory, and untuned inhibitory pads arrayed across multiple digits.

One possibility is that SII region neurons are responsible for coding local stimulus features and that neurons with untuned pads (types UE, UI, and UEI) serve to code tactile functions that are not orientation sensitive, such as texture (Sinclair and Burton, 1993; Jiang et al., 1997; Pruett et al., 2000, 2001), vibration (Poranen and Hyvarinen, 1982; Burton and Sinclair, 1991; Burton et al., 1997; Romo et al., 2002), or motion processing (Whitsel et al., 1972; Costanzo and Gardner, 1980; Warren et al., 1986; Ruiz et al.,1995), and that neurons with tuned pads (type T) are coding local oriented features such as Braille dots.

Another more likely possibility is that the RFs serve more integrative functions within the hand. Typically, we observed that, for individual neurons, the responsive pads are not randomly arrayed across the digits, suggesting that how those pads are organized plays an important role in their function. This is supported by the data showing the following: (1) the strongest responses usually occur on the distal finger pads, in particular on digits 2 and 3, which are the pads that are most frequently used in haptic exploration; (2) the responsive pads tend to be arrayed across separate digits rather than within digits, suggesting that those pads are involved in integrating information across the hand; and (3) the responsive pads tend to be clustered together in contiguous groups and are organized as structured combinations of excitatory and inhibitory subregions arrayed across the hand. These findings demonstrate that SII region RFs are highly organized and may be extracting features of large objects touched by the hand. Our working hypothesis is that these neurons form part of a set of kernels representing major structural components of objects. This is supported by evidence showing that the SII region is important for shape perception, because many neurons in the SII region are affected by proprioceptive input (Fitzgerald et al., 2004; Thakur et al., 2004), and lesion studies show that ablation of the SII region impairs recognition of object shape (Murray and Mishkin, 1984). Another major finding of this study is that many SII region neurons show orientation tuning on one or more finger pads (type T neurons), and most of these neurons contained additional untuned pads. Orientation feature selectivity has been shown to be important for representing object shape. Here we show that orientation tuning was commonly found in all three fields of the SII region. In a companion paper (Fitzgerald et al., 2006), we analyze these responses in more detail and show that many neurons are sharply tuned and that all orientations are well represented in the population response. Furthermore, we show that the preferred orientation tends to be similar across finger pads for neurons that had multiple tuned pads. This result suggests that SII region neurons may be important for coding both local and global features of objects.

There are various possibilities as to why some type T neurons had both tuned and untuned pads. One is that, in the current study, the oriented bar stimulus was not behaviorally relevant. Previous studies have shown that a large fraction of neurons in the SII region are affected by the animal's focus of attention (Poranen and Hyvarinen, 1982; Hsiao et al., 1993; Burton et al., 1997; Fitzgerald et al., 1998; Nakama et al., 2000; Meftah et al., 2002; Chapman and Meftah, 2005), and attending to the orientation of a bar results in enhancement of the tuning function of the neuron (Fitzgerald et al., 1998). This suggests that the RF characteristics we report here could change significantly under behavioral conditions in which the animal was required to attend to the bar. Therefore, these RFs may only be a rough estimate of the actual processing functions of the neurons, because the untuned pads may become tuned with attention. Another possibility is that these neurons are selective for objects with both edges and flat surfaces, such as the corner of a table.

A significant limitation of the results we present here is that, for all of the responsive neurons, it is not clear how stimuli presented to multiple pads simultaneously would interact because, in the current study, we only stimulated a single finger pad at a time. Is there response summation across finger pads for these large SII region RFs, and, if so, is such spatial summation linear or nonlinear? Summation in which stimulation of multiple finger pads evokes a greater response than stimulation of any individual finger pad (even if the observed sum is less than the linear sum) would be consistent with selectivity for large (multipad) objects, whereas lack of summation may indicate selectivity for small (single pad) objects. A previous study (Nakama et al., 2001) in which multiple digits were stimulated, both individually and simultaneously, with oriented bars indicates that many SII region RFs do exhibit spatial summation, although often it is less than the linear sum.

Nineteen percent of all SII region neurons studied were not responsive (type NR) to the oriented bar. The most likely explanation for this is that these neurons are responsive primarily to proprioceptive input (Thakur et al., 2004), have cutaneous responses that are modulated by hand conformation (Hsiao et al., 2002), or these neurons are processing information from nociceptive or thermal inputs (for review, see Peyron et al., 2000; Schnitzler and Ploner, 2000; Treede et al., 2000; Duncan and Albanese, 2003) (also see Whitsel et al., 1969; Robinson and Burton, 1980c; Porter, 1987).

\section{The SII region and the somatosensory cortical hierarchy}

The data presented in this paper are consistent with the hypothesis that the SII region comprises multiple representations (Fitzgerald et al., 2004), because the response properties of neurons in the three putative fields differed in many ways. A primary difference between neurons in the more cutaneously sensitive central field and those in the flanking posterior and anterior fields is unequal representation of the five RF types (Fig. 9), particularly types T and NR. This large increase in the proportion of neurons that are tuned in the central field and corresponding decrease in nonresponsive neurons suggests that this field may be particularly important for coding cutaneous object shape. The measures of RF size, shape, and somatotopy were somewhat similar for the three fields, although the central and anterior field RFs in Figures 12 and 13, as well as the relative location of the two most responsive pads analysis, were more similar to one another than they were to the posterior field RFs.

It is difficult to say whether the current data are more consistent with parallel processing within the SII region or instead with serial processing in which one representation provides feedforward input to another representation. The fact that the anterior and posterior fields show more similar proportions of neurons than either shows to the central field supports the hypothesis that the three fields process cutaneous information in parallel (Fitzgerald et al., 2004) and that the anterior and posterior fields code for features that depend on proprioceptive input.

Another general question about the SII region concerns its hierarchical relationship with respect to other somatosensory cortical areas. Here we have quantitatively demonstrated that 
most SII region hand RFs are multidigit, a finding that alone suggests it is of higher order than area $3 \mathrm{~b}$, for example, which primarily has single-digit RFs (Paul et al., 1972; Hyvarinen and Poranen, 1978b; Iwamura et al., 1983a; Chapman and Ageranioti-Belanger, 1991; DiCarlo et al., 1998). Its hierarchical relationship with areas $1,2,5,7 \mathrm{~b}$, retroinsula, and somatosensory areas of the insula is less clear based simply on RF size, because these areas also have multidigit RFs (Mountcastle et al., 1975; Robinson and Burton, 1980b; Iwamura et al., 1983b; Pons et al., 1985; Schneider et al., 1993).

\section{References}

Burton H, Sinclair RJ (1991) Second somatosensory cortical area in macaque monkeys. 2. Neuronal responses to punctate vibrotactile stimulation of glabrous skin on the hand. Brain Res 538:127-135.

Burton H, Fabri M, Alloway K (1995) Cortical areas within the lateral sulcus connected to cutaneous representations in areas $3 \mathrm{~b}$ and 1 : a revised interpretation of the second somatosensory area in macaque monkeys. J Comp Neurol 355:539-562.

Burton H, Sinclair RJ, Hong SY, Pruett JR, Whang KC (1997) Tactile-spatial and cross-modal attention effects in the second somatosensory and $7 \mathrm{~b}$ cortical areas of rhesus monkeys. Somatosens Mot Res 14:237-267.

Chapman CE, Meftah EM (2005) Independent controls of attentional influences in primary and secondary somatosensory cortex. J Neurophysiol 94:4094-4107.

Chapman CE, Ageranioti-Belanger SA (1991) Discharge properties of neurons in the hand area of primary somatosensory cortex in monkeys in relation to the performance of an active tactile discrimination task. Exp Brain Res 87:319-339.

Costanzo RM, Gardner EP (1980) A quantitative analysis of responses of direction-sensitive neurons in somatosensory cortex. J Neurophysiol 43:1319-1341.

DiCarlo JJ, Johnson KO (2000) Spatial and temporal structure of receptive fields in primate somatosensory area $3 \mathrm{~b}$ : effects of stimulus scanning direction and orientation. J Neurosci 20:495-510.

DiCarlo JJ, Lane JW, Hsiao SS, Johnson KO (1996) Marking microelectrode penetrations with fluorescent dyes. J Neurosci Methods 64:75-81.

DiCarlo JJ, Johnson KO, Hsiao SS (1998) Structure of receptive fields in area $3 \mathrm{~b}$ of primary somatosensory cortex of the alert monkey. J Neurosci 18:2626-2645.

Disbrow E, Litinas E, Recanzone GH, Padberg J, Krubitzer LA (2003) Cortical connections of the second somatosensory area and the parietal ventral area in macaque monkeys. J Comp Neurol 462:382-399.

Duncan GH, Albanese MC (2003) Is there a role for the parietal lobes in the perception of pain? Adv Neurol 93:69-86.

Fitzgerald PJ, Lane JW, Hsiao SS (1998) Attentional effects in somatosensory cortex during an orientation discrimination task. Soc Neurosci Abstr 24:444.11.

Fitzgerald PJ, Lane JW, Yoshioka T, Nakama T, Hsiao SS (1999) Multi-digit receptive field structures and orientation tuning properties of neurons in SII cortex of the awake monkey. Soc Neurosci Abstr 25:673.8.

Fitzgerald PJ, Lane JW, Thakur PH, Hsiao SS (2004) Receptive field properties of the macaque second somatosensory cortex: evidence for multiple functional representations. J Neurosci 24:11193-11204.

Fitzgerald PJ, Lane JW, Thakur PH, Hsiao SS (2006) Receptive field properties of the macaque second somatosensory cortex: representation of orientation on different finger pads. J Neurosci 26:6473-6484.

Friedman DP, Jones EG, Burton H (1980) Representation pattern in the second somatosensory area of the monkey cerebral cortex. JComp Neurol 192:21-41.

Friedman DP, Murray EA, O’Neill JB, Mishkin M (1986) Cortical connections of the somatosensory fields of the lateral sulcus of macaques: evidence for a corticolimbic pathway for touch. J Comp Neurol 252:323-347.

Hsiao SS, O'Shaughnessy DM, Johnson KO (1993) Effects of selective attention on spatial form processing in monkey primary and secondary somatosensory cortex. J Neurophysiol 70:444-447.

Hsiao SS, Pawluk D, Byrne A, Lane J (2002) Effects of changes in hand conformation on neural responses in areas $3 \mathrm{~b}, 1$, and 2 of the awake behaving monkey. Soc Neurosci Abstr 28:650.14.

Hyvarinen J, Poranen A (1978a) Movement-sensitive and direction and orientation-selective cutaneous receptive fields in the hand area of the post-central gyrus in monkeys. J Physiol (Lond) 283:523-537.

Hyvarinen J, Poranen A (1978b) Receptive field integration and submodality convergence in the hand area of the post-central gyrus of the alert monkey. J Physiol (Lond) 283:539-556.

Iggo A (1963) An electrophysiological analysis of afferent fibres in primate skin. Acta Neuroveg 24:225-240.

Iwamura Y, Tanaka M, Sakamoto M, Hikosaka O (1983a) Functional subdivisions representing different finger regions in area 3 of the first somatosensory cortex. Exp Brain Res 51:315-326.

Iwamura Y, Tanaka M, Sakamoto M, Hikosaka O (1983b) Converging patterns of finger representation and complex response properties of neurons in area 1 of the first somatosensory cortex of the conscious monkey. Exp Brain Res 51:327-337.

Jiang W, Tremblay F, Chapman CE (1997) Neuronal encoding of texture changes in the primary and the secondary somatosensory cortical areas of monkeys during passive texture discrimination. J Neurophysiol 77:1656-1662.

Krubitzer LA, Clarey J, Tweedale R, Elston G, Calford M (1995) A redefinition of somatosensory areas in the lateral sulcus of macaque monkeys. J Neurosci 15:3821-3839.

Meftah EM, Shenasa J, Chapman CE (2002) Effects of a cross-modal manipulation of attention on somatosensory cortical neuronal responses to tactile stimuli in the monkey. J Neurophysiol 88:3133-3149.

Mountcastle VB, Lynch JC, Georgopoulos A, Sakata H, Acuna C (1975) Posterior parietal association cortex of the monkey: command functions for operations within extrapersonal space. J Neurophysiol 38:871-908.

Murray EA, Mishkin M (1984) Relative contributions of SII and area 5 to tactile discrimination in monkeys. Behav Brain Res 11:67-83.

Nakama T, Lane JW, Fitzgerald PJ, Sripati A, Johnson KO, Yantis S, Hsiao SS (2000) Attentional modulation of bilateral neuronal responses in the secondary somatosensory cortex during an orientation discrimination task. Soc Neurosci Abstr 26:788.8.

Nakama T, Lane JW, Fitzgerald PJ, Johnson KO, Hsiao SS (2001) Linear regression analysis of bimanual responses of neurons to oriented bars in the second somatosensory cortex. Soc Neurosci Abstr 27:21.4.

Paul RL, Merzenich MM, Goodman H (1972) Representation of slowly and rapidly adapting cutaneous mechanoreceptors of the hand in Brodmann's areas 3 and 1 of Macaca mulatta. Brain Res 36:229-249.

Peyron R, Laurent B, Garcia-Larrea L (2000) Functional imaging of brain responses to pain. A review and meta-analysis. Neurophysiol Clin 30:263-288.

Pons TP, Garraghty PE, Cusick CG, Kaas JH (1985) The somatotopic organization of area 2 in macaque monkeys. J Comp Neurol 241:445-466.

Pons TP, Garraghty PE, Friedman DP, Mishkin M (1987) Physiological evidence for serial processing in somatosensory cortex. Science 237:417-420.

Pons TP, Garraghty PE, Mishkin M (1992) Serial and parallel processing of tactual information in somatosensory cortex of rhesus monkeys. J Neurophysiol 68:518-527.

Poranen A, Hyvarinen J (1982) Effects of attention on multiunit responses to vibration in the somatosensory regions of the monkey's brain. Electroencephalogr Clin Neurophysiol 53:525-537.

Porter LH (1987) An experimental investigation of the parietal lobes and temperature discrimination in monkeys. Brain Res 412:54-67.

Pruett JR, Sinclair RJ, Burton H (2000) Response patterns in second somatosensory cortex (SII) of awake monkeys to passively applied tactile gratings. J Neurophysiol 84:780-797.

Pruett JR, Sinclair RJ, Burton H (2001) Neural correlates for roughness choice in monkey second somatosensory cortex (SII). J Neurophysiol 86:2069-2080.

Robinson CJ, Burton H (1980a) Somatotopographic organization in the second somatosensory area of M. fascicularis. J Comp Neurol 192:43-67.

Robinson CJ, Burton H (1980b) Organization of somatosensory receptive fields in cortical areas 7b, retroinsula, postauditory, and granular insula of M. fascicularis. J Comp Neurol 192:69-92.

Robinson CJ, Burton H (1980c) Somatic submodality distribution within the second somatosensory (SII), 7b, retroinsular, postauditory, and granular insular cortical areas of M. fascicularis. J Comp Neurol 192:93-108.

Romo R, Hernandez A, Zainos A, Lemus L, Brody CD (2002) Neuronal correlates of decision-making in secondary somatosensory cortex. Nat Neurosci 5:1217-1225. 
Ruiz S, Crespo P, Romo R (1995) Representation of moving tactile stimuli in the somatic sensory cortex of awake monkeys. J Neurophysiol 73:525-537.

Schneider RJ, Friedman DP, Mishkin M (1993) A modality-specific somatosensory area within the insula of the rhesus monkey. Brain Res 621:116-120.

Schnitzler A, Ploner M (2000) Neurophysiology and functional neuroanatomy of pain perception. J Clin Neurophysiol 17:592-603.

Sinclair RJ, Burton H (1993) Neuronal activity in the second somatosensory cortex of monkeys (Macaca mulatta) during active touch of gratings. J Neurophysiol 70:331-350.

Talbot WH, Darian-Smith I, Kornhuber HH, Mountcastle VB (1968) The sense of flutter-vibration: comparison of the human capacity with response patterns mechanoreceptive afferents from the monkey hand. J Neurophysiol 31:301-334.
Thakur PH, Fitzgerald PJ, Byrne AH, Pembeci I, Berryman LJ, Hsiao SS (2004) Neural responses to changes in hand conformation in the second somatosensory cortex. Soc Neurosci Abstr 30:59.10.

Treede R, Apkarian AV, Bromm B, Greenspan JD, Lenz FA (2000) Cortical representation of pain: functional characterization of nociceptive areas near the lateral sulcus. Pain 87:113-119.

Warren S, Hamalainen HA, Gardner EP (1986) Objective classification of motion- and direction-sensitive neurons in primary somatosensory cortex of awake monkeys. J Neurophysiol 56:598-622.

Whitsel BL, Petrucelli LM, Werner G (1969) Symmetry and connectivity in the map of the body surface in somatosensory area II of primates. J Neurophysiol 32:170-182.

Whitsel BL, Roppolo JR, Werner G (1972) Cortical information processing of stimulus motion on primate skin. J Neurophysiol 35:691-717. 